\title{
Multistate ring polymer instantons and nonadiabatic reaction rates
}

\author{
Srinath Ranya ${ }^{1}$ and Nandini Ananth 1 , a) \\ Department of Chemistry and Chemical Biology, Cornell University, Ithaca, New York, 14853, \\ $U S A$
}

(Dated: 27 February 2020)

We present two multistate ring polymer instanton (RPI) formulations, both obtained from an exact path integral representation of the quantum canonical partition function for multistate systems. The two RPIs differ in their treatment of the electronic degrees of freedom; whereas the Mean-Field (MF)-RPI averages over the electronic state contributions, the Mapping Variable (MV)-RPI employs explicit continuous Cartesian variables to represent the electronic states.

We compute both RPIs for a series of model two-state systems coupled to a single nuclear mode with electronic coupling values chosen to describe dynamics in both adiabatic and nonadiabatic regimes. We show that the MF-RPI for symmetric systems are in good agreement with previous literature, and we show that our numerical techniques are robust for systems with non-zero driving force. The nuclear MF-RPI and the nuclear MV-RPI are similar, but the MV-RPI uniquely reports on the changes in the electronic state populations along the instanton path. In both cases, we analytically demonstrate the existence of a zero-mode and we numerically find that these solutions are true instantons with a single unstable mode as expected for a first order saddle point. Finally, we use the MF-RPI to accurately calculate rate constants for adiabatic and nonadiabatic model systems with the coupling strength varying over three orders of magnitude.

Keywords: Nonadiabatic instanton, mapping variables, ring polymer instanton, mean-field, two-state system

\section{INTRODUCTION}

Nonadiabatic charge and energy transfer processes are the key step in the functioning of many biological and chemical systems $\stackrel{1}{-5}^{-5}$ As such, the computation of nonadiabatic reaction rates has been the subject of a great deal of interest for over two decades, leading to the development of several nonadiabatic dynamic approaches. An alternate low-cost strategy to compute rates for adiabatic and nonadiabatic processes is the computation of so-called 'instantons' ${ }^{6.7}$ An instanton is a periodic orbit in imaginary time on an inverted potential energy surface $^{8}$ and is, typically, the trajectory that contributes most to the flux-side correlation function. $\underline{9,10}$

Semiclassical instanton rate theory has been employed with a great deal of success for the computation of adiabatic reaction rates $11-14$ More recently, the ring polymer instanton (RPI) method based on the path integral formulation of quantum mechanics has been developed ${ }^{15}$ and it's connections with semiclassical theory established. ${ }^{16,17}$ Early work towards nonadiabatic rates via an instanton formulation extended the semiclassical instanton approach to multistate systems and was successfully used to calculate both adiabatic and nonadiabatic rates for symmetric systems $18-20$ Other methods to compute instanton rates valid only in the nonadiabatic or weak-coupling limit include a saddle point approximation to the flux-flux correlation function by Wolynes, ${ }^{21}$ recently shown to be accurate in the inverted Marcus regime $;^{22}$ and a nonadiabatic instanton obtained by extending Gutzwiller's work ${ }^{23,24}$ to imaginary time

a) Electronic mail: ananth@cornell.edu. and energy-matching two single surface instantons at the point of crossing. 25,26

In this paper, we numerically calculate multistate RPIs that are first order saddles, we obtain analytic expressions for the zero mode, and we derive an expression to calculate rate constants applicable to both nonadiabatic and adiabatic processes. We derive two expressions for multistate RPIs. The first, a Mean-Field (MF)-RPI, is obtained by finding the stationary path in imaginary time from the exact mean-field path integral representation of the quantum canonical partition function. The MF-RPI closely follows previously proposed nonadiabatic instantons $^{18}$ - it is accurate for both nonadiabatic and adiabatic processes but does not explicitly report on transitions between electronic states along the instanton path. An equivalent representation of the canonical partition function of multistate systems can be obtained employing continuous Cartesian variables for both the nuclear degrees of freedom and the electronic state variables using the Meyer-Miller-Stock-Thoss mapping protocol ${ }^{27-29}$ Such a mapping-variable (MV) formulation has been shown to be particularly useful in developing approximate nonadiabatic dynamic methods $30-34$ Here, we compute the continuous mapping-variable (MV)-RPI that explicitly includes both nuclear positions and electronic state populations along the instanton path.

We numerically compute the MF-RPI and the MVRPI for a series of model two-state systems coupled to a single nuclear degree of freedom using the Limitedmemory Broyden-Fletcher-Goldfarb-Shanno minimization algorithm with box constraints (L-BFGS-B) ${ }^{35}$ We show that both nuclear instantons correctly describe the physics of a nonadiabatic transition. We further demonstrate that the MV-RPI uniquely reports on changes in electronic state populations in both the adiabatic and 
nonadiabatic regimes without any assumptions about the nuclear positions at which electronic state transitions occur. Finally, we derive an MF-RPI rate expression and show that the resulting rate constants agree well with Fermi's Golden Rule (FGR) rates for nonadiabatic model systems and with single surface RPI rates in the adiabatic regime.

The paper is organized is as follows: In Sec. II we provide an overview of the MF-RPI and the MV-RPI and introduce the MF-RPI rate expression. We provide a brief description of the model systems in Sec. III. Implementation details are provided in Sec. IV and we present our results and conclusions in Sec. V] and Sec. VI, respectively.

\section{THEORY}

\section{A. Single Surface Ring Polymer Instanton}

In general, the Hamiltonian corresponding to a single surface system with $f$ nuclear degrees of freedom (dofs) can be written as

$$
\hat{H}(\hat{\mathbf{R}}, \hat{\mathbf{P}}) \equiv \frac{\hat{\mathbf{P}}^{T} \hat{\mathbf{P}}}{2 M}+\mathrm{V}(\hat{\mathbf{R}}),
$$

where, $\hat{\mathbf{R}}$ and $\hat{\mathbf{P}}$ represent the positions and momenta of the nuclear dofs, $M$ is the nuclear mass, and $\mathrm{V}(\hat{\mathbf{R}})$ is the potential. The path integral expression for the quantum canonical partition function is obtained from the trace of the Boltzmann operator by inserting multiple copies of identity in the form of a complete set of nuclear position states,

$$
\begin{aligned}
\mathcal{Z} & =\operatorname{Tr}\left[e^{-\beta \hat{H}}\right] \\
& \propto \lim _{N \rightarrow \infty} \int d\left\{\mathbf{R}_{\alpha}\right\} e^{-\beta V_{\mathrm{RP}}},
\end{aligned}
$$

where we omit pre-multiplicative constants, $\beta=1 / k_{B} T$ and we use the notation $\int d\left\{\mathbf{R}_{\alpha}\right\} \equiv \int d \mathbf{R}_{1} \ldots \int d \mathbf{R}_{N}$. In Eq. 2, we define the isomorphic ring polymer potential as $V_{\mathrm{RP}}=U\left(\left\{\mathbf{R}_{\alpha}\right\}\right)+(1 / N) \sum_{\alpha} \mathrm{V}\left(\mathbf{R}_{\alpha}\right)$, where the inter-bead interaction potential is

$$
U\left(\left\{\mathbf{R}_{\alpha}\right\}\right)=\frac{1}{N} \sum_{\alpha} \frac{M}{2 \beta_{N}^{2}}\left(\mathbf{R}_{\alpha}-\mathbf{R}_{\alpha+1}\right)^{T}\left(\mathbf{R}_{\alpha}-\mathbf{R}_{\alpha+1}\right),
$$

and $\beta_{N}=\beta / N$

The RPI is a discretized approximation to the instanton path, and is a first order saddle point on the ring polymer potential $\frac{15}{1 t}$ is determined by setting the gradient of the isomorphic classical potential $V_{\mathrm{RP}}$ to zero,

$$
\frac{\partial V_{\mathrm{RP}}}{\partial\left[\mathbf{R}_{\alpha}\right]_{i}}=0
$$

where bead index $\alpha=1 \ldots N$ and the nuclear dofs are indexed by $i=1 \ldots f$. In the absence of an analytic solu- tion, the RPI is numerically obtained as the simultaneous solution of the $f N$ equations in Eq. 4.

We note that the ring polymer potential, $V_{\mathrm{RP}}$, is invariant to cyclic permutation of the beads that define the RPI path and in the $N \rightarrow \infty$ it reproduces the familiar result — the invariance of the instanton path action to imaginary time translation.

\section{B. Canonical Partition Function for Multistate Systems}

The potential for a multi-state system with $\mathcal{K}$ electronic states and $f$ nuclear dofs in the diabatic representation is,

$$
\mathbf{V}(\hat{\mathbf{R}})=\sum_{n, m=1}^{\mathcal{K}}\left|\psi_{n}\right\rangle V_{n m}(\hat{\mathbf{R}})\left\langle\psi_{m}\right|
$$

where $\left\{\left|\psi_{n}\right\rangle\right\}$ are the diabatic electronic states, the diagonal matrix elements $V_{n n}(\hat{\mathbf{R}})$ are the potentials corresponding to the $n^{\text {th }}$ state, and the off-diagonal matrix elements $V_{n m}(\hat{\mathbf{R}})$ describe the electronic couplings between states $n$ and $m$. The canonical partition function is expressed as a trace over the Boltzmann operator,

$$
\begin{aligned}
\mathcal{Z} & =\operatorname{Tr}_{n e}\left[e^{-\beta \hat{H}}\right] \\
& \propto \lim _{N \rightarrow \infty} \int d\left\{\mathbf{R}_{\alpha}\right\} e^{-\beta U\left(\left\{\mathbf{R}_{\alpha}\right\}\right)} \operatorname{Tr}_{e}\left[\prod_{\alpha=1}^{N} e^{-\beta_{N} \mathbf{V}\left(\mathbf{R}_{\alpha}\right)}\right](6)
\end{aligned}
$$

where the subscripts $n$ and $e$ indicate that the trace is evaluated over the nuclear and electronic dofs respectively, and $U\left(\left\{\mathbf{R}_{\alpha}\right\}\right)$ is the inter-bead potential previously defined in Eq. 3. We obtain Eq. 6] by evaluating the trace over the nuclear dofs in the position basis. The trace over electronic dofs can be evaluated in a number of ways; $29,32,33,36,37$ here we explore the MF and one MV formulation with explicit electronic state variables.

\section{Mean-Field Representation}

The MF representation of the canonical partition function $18,19,32,37-39$ is obtained by introducing $N$ copies of the identity,

$$
\mathbb{1}=\sum_{n}\left|\psi_{n}\right\rangle\left\langle\psi_{n}\right|
$$

in Eq. 6] and evaluating the trace to obtain 40

$$
\operatorname{Tr}_{e}\left[\prod_{\alpha=1}^{N} e^{-\beta_{N} \mathbf{V}\left(\mathbf{R}_{\alpha}\right)}\right]=\operatorname{Tr}\left[\prod_{\alpha=1}^{N} \mathcal{M}\left(\mathbf{R}_{\alpha}, \mathbf{R}_{\alpha+1}\right)\right] \equiv \Gamma_{\mathrm{MF}}
$$


where the matrix elements $\mathcal{M}\left(\mathbf{R}_{\alpha}, \mathbf{R}_{\alpha+1}\right)$ are

$$
\begin{aligned}
\mathcal{M}_{n n} & =e^{-\beta_{N} / 2\left[V_{n n}\left(\mathbf{R}_{\alpha}\right)+V_{n n}\left(\mathbf{R}_{\alpha}\right)\right]}, \\
\mathcal{M}_{n m} & =-\beta_{N} / 4\left[V_{n m}\left(\mathbf{R}_{\alpha}\right)+V_{n m}\left(\mathbf{R}_{\alpha+1}\right)\right] \\
& \times\left[\begin{array}{c}
e^{-\beta_{N} / 2\left[V_{n n}\left(\mathbf{R}_{\alpha}\right)+V_{n n}\left(\mathbf{R}_{\alpha+1}\right)\right]} \\
+e^{-\beta_{N} / 2\left[V_{m m}\left(\mathbf{R}_{\alpha}\right)+V_{m m}\left(\mathbf{R}_{\alpha+1}\right)\right]}
\end{array}\right] .
\end{aligned}
$$

The quantum canonical partition function in the MF representation is then

$$
\mathcal{Z}_{\mathrm{MF}} \propto \lim _{N \rightarrow \infty} \int d\left\{\mathbf{R}_{\alpha}\right\} e^{-\beta V_{\mathrm{MF}}\left(\left\{\mathbf{R}_{\alpha}\right\}\right)} \operatorname{sgn}\left(\Gamma_{\mathrm{MF}}\right)
$$

where $\operatorname{sgn}\left(\Gamma_{\mathrm{MF}}\right)$ ensures that the partition function is positive definite, and we have omitted pre-multiplicative constants. The effective MF ring polymer potential is

$$
V_{\mathrm{MF}}\left(\left\{\mathbf{R}_{\alpha}\right\}\right)=U\left(\left\{\mathbf{R}_{\alpha}\right\}\right)-\frac{1}{\beta} \ln \left|\operatorname{Re}\left(\Gamma_{\mathrm{MF}}\right)\right|,
$$

where $\Gamma_{\mathrm{MF}}$, given in Eq. 8, averages over the electronic state configurations of the ring polymer making this a 'mean-field' formulation.

\section{Mapping Variable Representation}

We introduce continuous Cartesian variables for the electronic states using the MMST mapping proto$\operatorname{col}^{27,28,41}$ Specifically, the $\mathcal{K}$ diabatic electronic states are mapped to a singly excited oscillator (SEO) basis where $\mathcal{K}-1$ harmonic oscillators are in the ground state and one oscillator (the $n^{\text {th }}$ ) is in the first excited state,

$$
\begin{aligned}
\left|\psi_{n}\right\rangle\left\langle\psi_{m}\right| & \rightarrow \hat{a}_{n}^{\dagger} \hat{a}_{m} \\
\left|\psi_{n}\right\rangle & \rightarrow\left|0_{1}, \ldots, 1_{n}, \ldots, 0_{\mathcal{K}}\right\rangle \equiv|n\rangle
\end{aligned}
$$

The resolution of identity in the electronic variables $(\mathbf{x})$ is 29

$$
\mathbb{1}=\int d \mathbf{x}|\mathbf{x}\rangle\langle\mathbf{x}| \mathcal{P}
$$

where the projection operator $\mathcal{P}=\sum_{n}|n\rangle\langle n|$ constrains the electronic coordinates to the SEO subspace.

Introducing multiple copies of this identity and evaluating the electronic trace in Eq. 6, we obtain an expression for the partition function: ${ }^{29}$

$$
\mathcal{Z}_{\mathrm{MV}} \propto \lim _{N \rightarrow \infty} \int d\left\{\mathbf{R}_{\alpha}\right\} \int d\left\{\mathbf{x}_{\alpha}\right\} e^{-\beta V_{\mathrm{MV}}} \operatorname{sgn}\left(\Gamma_{\mathrm{MV}}\right),
$$

where

$$
\Gamma_{\mathrm{MV}}=\operatorname{Tr}\left[\prod_{\alpha=1}^{N} \mathcal{X}_{\alpha} \mathcal{M}\left(\mathbf{R}_{\alpha}, \mathbf{R}_{\alpha+1}\right)\right]
$$

the matrix $\mathcal{X}_{\alpha}=\mathbf{x}_{\alpha} \otimes \mathbf{x}_{\alpha}^{T}$, and the MV ring polymer potential is,

$$
V_{\mathrm{MV}}\left(\left\{\mathbf{R}_{\alpha}\right\}\right)=U\left(\left\{\mathbf{R}_{\alpha}\right\}\right)+\frac{1}{\beta} \sum_{\alpha} \mathbf{x}_{\alpha}^{T} \mathbf{x}_{\alpha}-\frac{1}{\beta} \ln \left|\operatorname{Re}\left(\Gamma_{\mathrm{MV}}\right)\right| .
$$

\section{Multistate Ring Polymer Instanton}

The multistate (MS) RPI is obtained by finding the $\mathrm{MF}$ or MV ring polymer configuration that is a first order saddle point on the corresponding potential energy surface. In the MF-RP formulation, the $f N$ equations that must be solved simultaneously are

$$
\frac{\partial V_{\mathrm{MF}}}{\partial\left[\mathbf{R}_{\alpha}\right]_{i}}=0
$$

where $\alpha$ is the bead number index and index $i$ runs over the nuclear dofs. The MV-RPI is obtained by solving $(f+\mathcal{K}) N$ equations simultaneously

$$
\begin{aligned}
& \frac{\partial V_{\mathrm{MV}}}{\partial\left[\mathbf{R}_{\alpha}\right]_{i}}=0 \text { and } \\
& \frac{\partial V_{\mathbf{M V}}}{\partial\left[\mathbf{x}_{\alpha}\right]_{j}}=0
\end{aligned}
$$

where the index $j$ runs over the electronic states.

\section{Zero mode of the instanton}

A true instanton solution is a first order saddle with one negative eigenvalue and a zero eigenvalue due to the invariance of the action under imaginary time translation. 11 In the case of the single surface RPI, the zero eigenvalue mode corresponds to the invariance of the isomorphic ring polymer potential under cyclic permutation of the beads. ${ }^{15}$ Here, we establish the existence of a negative eigenvalue numerically for both the MF-RPI and MV-RPI, and we analytically find the zero mode in each case. The action for multistate systems, $\mathcal{S}_{\mathrm{MS}}$, is obtained by taking the continuum limit of the isomorphic ring polymer potential, $V_{\mathrm{MS}}$, where $\mathrm{MS}=\{\mathrm{MF}, \mathrm{MV}\}$,

$$
\mathcal{S}_{\mathrm{MS}}=\int_{0}^{\beta} d \tau\left[\frac{M}{2}\left(\frac{d \mathbf{X}(\tau)}{d \tau}\right)^{2}+\mathrm{V}_{\mathrm{MS}}[\mathbf{X}(\tau)]\right]
$$

and $\mathbf{X}(\tau)$ is a vector of all the dofs (nuclear only for the MF-RPI, and nuclear and electronic for the MV-RPI) in imaginary time, $\tau$. The MS-instanton solution is obtained by setting the first variation of the action to zero,

$$
\delta \mathcal{S}_{\mathrm{MS}}=-M \frac{d^{2} \mathbf{X}(\tau)}{d \tau^{2}}+\nabla_{\mathbf{X}} \mathrm{V}_{\mathrm{MS}}[\mathbf{X}(\tau)]=0
$$

The second variation of the action, $\delta^{2} \mathcal{S}_{\mathrm{MS}}$, is the stability matrix $\left(\Lambda_{\mathrm{MS}}\right)$ which incorporates the effects of path 
fluctuations about the MS instanton,

$$
\begin{aligned}
\delta^{2} \mathcal{S}_{\mathrm{MS}} & =\int_{0}^{\beta} d \tau \delta \mathbf{X}(\tau)^{T} \Lambda_{\mathrm{MS}} \delta \mathbf{X}(\tau) \\
\Lambda_{\mathrm{MS}} & =\left[-M \frac{d^{2}}{d \tau^{2}}+\nabla_{\mathbf{X}}^{T} \nabla_{\mathbf{X}} \mathrm{V}_{\mathrm{MS}}\right]
\end{aligned}
$$

Differentiating Eq. 21 with respect to imaginary time,

$$
\frac{d}{d \tau}\left[-M \frac{d^{2} \mathbf{X}(\tau)}{d \tau^{2}}+\nabla_{\mathbf{X}} \mathrm{V}_{\mathrm{MS}}\right]=\Lambda_{\mathrm{MS}} \dot{\mathbf{X}}(\tau)=0 \times \dot{\mathbf{X}}(\tau)
$$

we find the zero-mode of the MS-instanton corresponds to a velocity mode in all the system dofs. Details of the derivation along with the stability matrices corresponding to the MF-RPI and MV-RPI are provided in Appendices $\mathrm{A}$ and $\mathrm{B}$, respectively.

\section{E. Reaction Rate from MF-RPI}

We can express the MF RPI rate as $15,16,42$

$$
\begin{aligned}
k_{\mathrm{MF}-\mathrm{RPI}} & \approx \frac{2}{\beta} \frac{\mathcal{Z}_{b}}{\mathcal{Z}_{r}} \\
& =\frac{2 e^{-\beta_{N} V_{\mathrm{MF}}\left(\tilde{\mathbf{R}}_{\alpha}\right)}}{\mathcal{Z}_{r} \beta}\left(\frac{M}{2 \pi \beta_{N}}\right)^{\frac{N}{2}} \\
& \times \int d\left\{\mathbf{R}_{\alpha}\right\} e^{-\beta_{2 N} V_{\mathrm{MF}}^{\prime \prime}\left(\tilde{\mathbf{R}}_{\alpha}\right)\left(\mathbf{R}_{\alpha}-\tilde{\mathbf{R}}_{\alpha}\right)^{2}},
\end{aligned}
$$

where $\left\{\tilde{\mathbf{R}}_{\alpha}\right\}$ represents the MF-RPI configuration, $\mathcal{Z}_{b}$ is the barrier partition function, $\mathcal{Z}_{r}$ is the reactant partition function, and the second derivative $V_{\mathrm{MF}}^{\prime \prime}$ is evaluated at the MF-RPI configuration. In Eq. 25, the last line is obtained by Taylor expanding the MF-PES about the MF-RPI solution and truncating to second order. The diagonalization of the hessian $V_{\mathrm{MF}}^{\prime \prime}\left(\left\{\tilde{\mathbf{R}}_{\alpha}\right\}\right)$ yields $N$ eigenvalues $\left(M \lambda_{\alpha}^{2}\right)$ :

$$
\begin{aligned}
k_{\mathrm{MF}-\mathrm{RPI}} & =\frac{2 e^{-\beta_{N} V_{\mathrm{MF}}\left(\tilde{\mathbf{R}}_{\alpha}\right)}}{\mathcal{Z}_{r} \beta}\left(\frac{M}{2 \pi \beta_{N}}\right)^{\frac{N}{2}} \\
& \times \int d\left\{\mathbf{s}_{\alpha}\right\} e^{-\beta_{2 N} \sum_{\alpha} M \lambda_{\alpha}^{2} \mathbf{s}_{\alpha}^{2}}
\end{aligned}
$$

The Hessian evaluated at the MF-RPI configuration has one negative eigenvalue, $\lambda_{1}<0$, and a zero eigenvalue, $\lambda_{2} \approx 0$, for a bead-converged calculation. We use this to evaluate the integral in Eq. 26 - mode $\mathbf{s}_{1}$ is integrated by analytically continuing the Gaussian and performing the integral over the positive part of the imaginary axis, mode $\mathbf{s}_{2}$ is integrated out analytically as are the remaining $(N-2)$ Gaussian integrals. We note that this derivation closely follows that employed in deriving the single surface RPI rate $\stackrel{15}{ }$ The resulting expression for the MF-
$\mathrm{RPI}$ rate constant is then,

$$
k_{\mathrm{MF}-\mathrm{RPI}} \approx \frac{e^{-\beta_{N} V_{\mathrm{MF}}\left(\tilde{\mathbf{R}}_{\alpha}\right)}}{\mathcal{Z}_{r} \beta_{N}}\left(\frac{M z_{N}}{2 \pi \beta_{N}}\right)^{\frac{1}{2}} \prod_{\alpha=1}^{N} \frac{1}{\beta_{N}\left|\lambda_{\alpha}\right|},
$$

where the ' on the product indicates that $\alpha=2$ is excluded, and $z_{N}=\sum_{\alpha}\left(\tilde{\mathbf{R}}_{\alpha}-\tilde{\mathbf{R}}_{\alpha+1}\right)^{2}$. This expression follows a previously derived nonadiabatic instanton rate, with the primary difference being the method used to find the instanton. 18

\section{MODEL SYSTEMS}

We find the multistate RPI for model systems with two electronic states $(\mathcal{K}=2)$ coupled to one nuclear dof $(f=1)$, and with three different driving forces. We note that all model systems are in the normal regime of Marcus theory where the protocol described here yields converged instanton solutions. Diagonal elements of the diabatic potential energy matrix, $\mathbf{V}(R)$, are

$$
V_{i i}(R)=\frac{1}{2} M \omega^{2}\left(R-R_{i}\right)^{2}+\epsilon \delta_{1 i},
$$

where $i=\{1,2\}$, the nuclear mass $M=2$ a.u., the oscillator frequency $\omega=1$ a.u. In Eq. 28, the Kronecker delta, $\left(\delta_{1 i}\right)$, indicates that a driving-force $\epsilon=0.0,10.0,20.0$ a.u. is added to the donor state (left curve), as shown in Fig.1. We choose $\beta>\beta_{c}$ such that an instanton solution exists

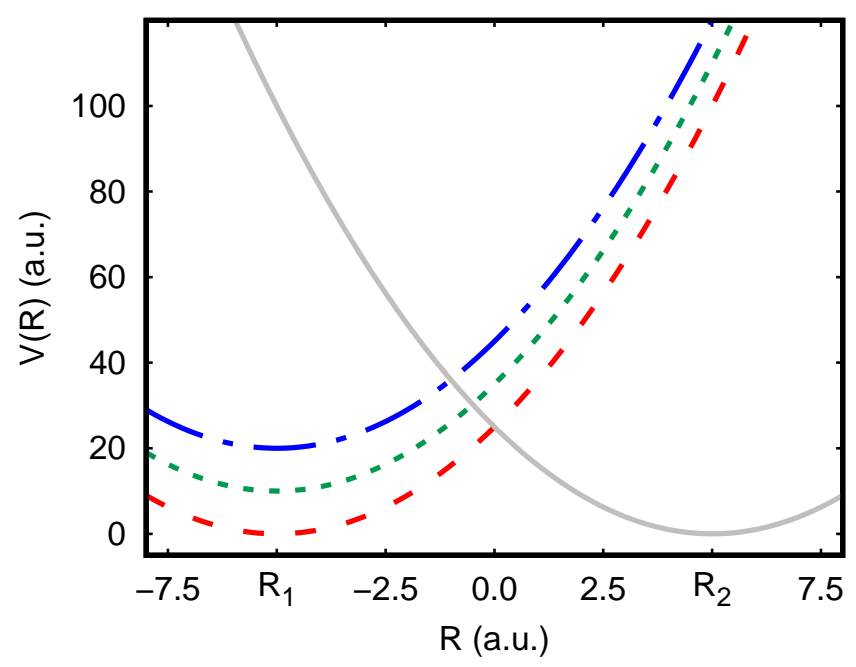

FIG. 1. Red (dashed) and grey (continuous) lines show the donor and acceptor states in model I (symmetric system); the green (dotted) and black (dot-dashed) lines represent the donor state in models II and III which are asymmetric systems with $\epsilon=10.0$ a.u. and 20.0 a.u., respectively. $R_{1}$ and $R_{2}$ indicate the minima of the donor and acceptor states.

in the adiabatic limit; we note that there is no clear analytical expression for the nonadiabatic crossover temperature ${ }^{20}$ For the systems considered here, in the adiabatic 
limit ( $\Delta=5$ a.u.) we obtain a barrier frequency $\omega_{b} \approx 3$ a.u. by fitting to an inverted parabola, and we use this to find $\beta_{c}=2 \pi / \omega_{b} \approx 2$ a.u. for all models $\stackrel{42}{\underline{42}}$ The electronic coupling and inverse temperature $(\beta)$ values used here are reported in Table. []

\begin{tabular}{|c|c|c|}
\hline$\beta$ & Adiabatic $\Delta$ & Nonadiabatic $\Delta$ \\
\hline 4.0 & 5.0 & $6.25 \mathrm{E}-3$ \\
3.75 & 5.33 & $6.67 \mathrm{E}-3$ \\
3.5 & 5.71 & $7.14 \mathrm{E}-3$ \\
3.25 & 6.15 & $7.70 \mathrm{E}-3$ \\
\hline
\end{tabular}

TABLE I. Parameters for models I, II, and III, chosen such that $\beta \Delta$ remains constant. All values are in atomic units.

\section{SIMULATION DETAILS}

\section{A. Optimization Algorithm}

We use the L-BFGS-B algorithm to solve the simultaneous equations for the instanton 35 The L-BFGS-B algorithm is an efficient, quasi-Newton approach to optimization and the box constraints allow for each variable in the search to have both an upper and lower bound. We find that a physically reasonable initial guess allows us to converge to a true instanton solution.

We use the following protocol to generate initial configurations. The minima of the two diabatic states are used to provide an upper and lower bound for the nuclear bead positions, and two beads are fixed at the crossing between the diabatic surfaces. We find both these constraints necessary to ensure we obtain an RPI solution that is a first order saddle rather than a minimum. The electronic coordinates are constrained to lie between -1 and 1 in all our calculations, but we find that changing this does not affect the outcome of the optimization.

In addition to the nuclear and electronic variables, it is necessary to also optimize the ratio of number of beads to the left and right of the crossing, $N_{1} / N_{2}$. Rather than incorporating this as an additional variable in our optimization algorithm, we fix this ratio for each calculation. The value of $N_{1} / N_{2}$ that maximizes the isomorphic ring polymer potential is the multistate RPI solution as shown in Fig. 2.

\section{B. Initial guess for the nuclear and electronic coordinates}

The optimization algorithm to find the RPI requires an initial guess. In theory the outcome should be independent of this guess, however, we find that a good initial guess is necessary to obtain a converged instanton solution for the large number of dofs in these calculations. Following previous work $\stackrel{26}{\underline{n}}$ we find it necessary to

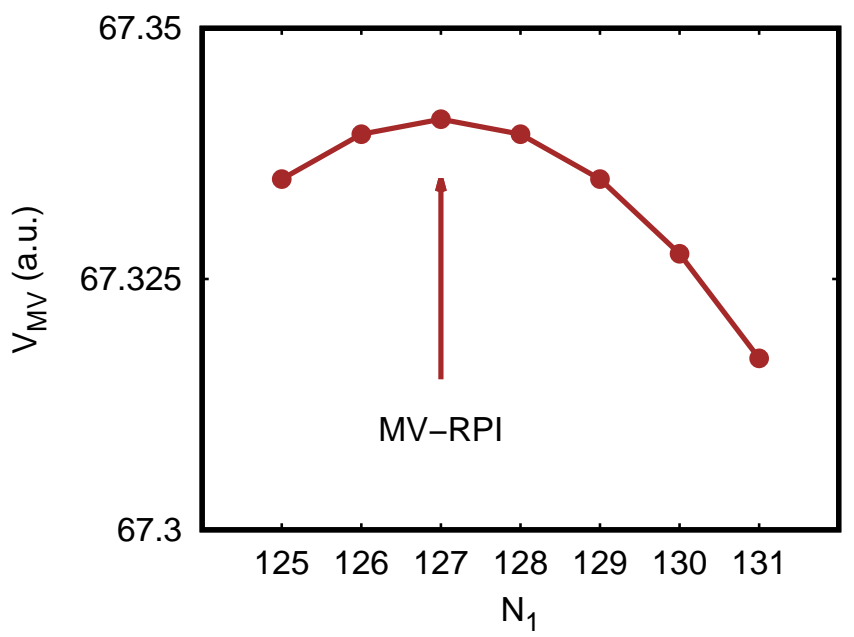

FIG. 2. The effective mapping variable ring polymer potential energy for a 256-bead ring polymer as a function of the number of beads to the left of the crossing $\left(N_{1}\right)$. The system shown here is symmetric model I with $\beta=3.25$ a.u. in the nonadiabatic regime, $\Delta=0.0077$ a.u. The RPI configurations corresponds to the maximum where $N_{1}=N_{2}=127$ beads $(2$ beads are fixed at the crossing).

fix two beads to the crossing point particularly for models with non-zero driving force where an unconstrained initial guess leads to a solution where all the ring polymer beads are in the lower-energy acceptor state rather than a first order saddle. We initialize $N_{1}$ beads to the left (donor state) and $N_{2}$ beads to the right (acceptor state) of the crossing, respectively, such that the total number of beads of the ring polymer $N=N_{1}+N_{2}+2$.

We begin by generating initial conditions for the nuclear positions of the RPI according to the equations,

$$
\begin{aligned}
& R_{j}=R_{0}+\left(R_{2}-R_{0}\right) \cos \left(\frac{\pi i}{N_{2}+3}\right) \\
& \quad \text { where } i=1,\left\lfloor\frac{N_{2}}{2}\right\rfloor+1, j=1,\left\lfloor\frac{N_{2}}{2}\right\rfloor+1 \\
& R_{\left\lfloor N_{2} / 2\right\rfloor+2}=R_{0} \\
& R_{j}=R_{0}-\left\lfloor\left(R_{0}-R_{1}\right) \cos \left(\frac{\pi}{2}+\frac{\pi i}{N_{1}+1}\right) \mid\right. \\
& \quad \text { where } i=1, N_{1}, j=\left\lfloor\frac{N_{2}}{2}\right\rfloor+3,\left\lfloor\frac{N_{2}}{2}\right\rfloor+N_{1}+2 \\
& \left.R_{\left\lfloor N_{2} / 2\right\rfloor+N_{1}+3=R_{0}}\right\rfloor \\
& R_{j}=R_{0}+\left(R_{2}-R_{0}\right) \cos \left(\frac{\pi i}{N_{2}+3}\right) \\
& \quad \text { where } i=\left\lfloor\frac{N_{2}}{2}\right\rfloor+4, N_{2}+2, j=\left\lfloor\frac{N_{2}}{2}\right\rfloor+N_{1}+4, N
\end{aligned}
$$

Note that in the equations above, we take the lowest integer for $N_{2} / 2$.

As a first step, for each model, we find the single surface RPI on the lower adiabatic surface. We use the nu- 
clear positions from this RPI as the initial guess for our MF-RPI calculation in the adiabatic limit. The MF-RPI solution, in turn, provides initial nuclear configurations for the MV-RPI calculation, and we estimate initial values for the electronic coordinates from the nuclear coordinates of the single surface instanton,

$$
\left[\mathbf{x}_{\alpha}\right]_{n}=\sqrt{\frac{e^{-\beta V_{n n}\left(R_{\alpha}\right)}}{\sum_{n} e^{-\beta V_{n n}\left(R_{\alpha}\right)}}}
$$

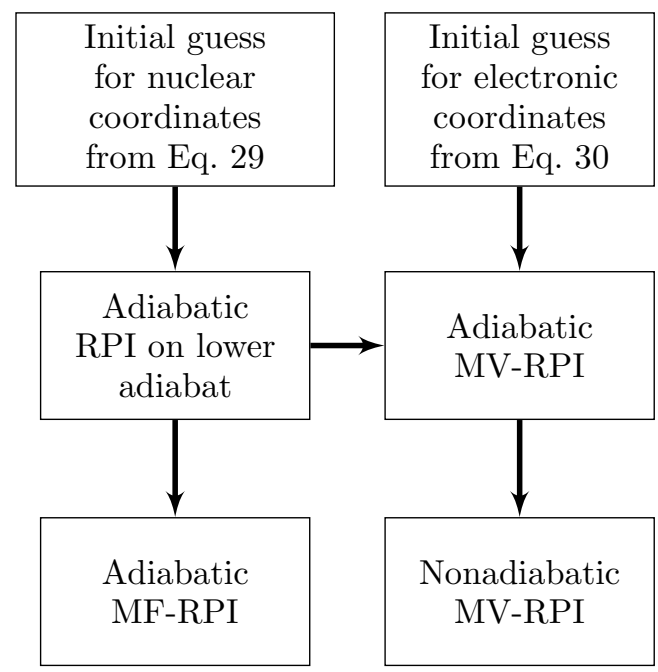

FIG. 3. We start with an initial nuclear configuration generated according to Eq. 29 and find the single surface RPI on the lower adiabatic state. The initial electronic configurations are generated according to Eq. 30. The arrows connect initial guess configurations with optimized RPI configurations.

The flowchart in Fig. 3 outlines the procedure used to generate initial configurations where we use the fact that the single surface RPI, the MF-RPI, and the MVRPI should have identical nuclear configurations in the adiabatic limit. The nonadiabatic RPI calculations employ the adiabatic RPIs as the initial guess for nuclear configurations in all cases.

\section{Nonadiabatic and Adiabatic Reaction Rate Theories}

For a two-level system where the reactant and product potential surfaces are displaced harmonic oscillators, the nonadiabatic reaction rate according to Fermi's Golden Rule (FGR) rate is

$$
k_{\mathrm{FGR}}=\frac{2 \pi}{\omega}|\Delta|^{2} e^{\nu \mathrm{z}-S \operatorname{coth}(\mathrm{z})} I_{\nu}(S \operatorname{csch}(\mathrm{z})),
$$

where $\mathrm{z}=\beta \omega / 2, \nu=\epsilon / \omega, S=M \omega\left(R_{r}-R_{p}\right)^{2} / 2$, and $I_{\nu}$ is a modified Bessel function of the first kind. $R_{r}$ and $R_{p}$ are minima of the reactant and product oscillators, and $\omega$ is their frequency.

In the adiabatic limit, the single surface RPI rate $\underline{\underline{15}}$ on the lower adiabat is:

$$
k_{\mathrm{RP}} \approx \frac{e^{-\beta_{N} V_{\mathrm{RP}}\left(\tilde{\mathbf{R}}_{\alpha}\right)}}{\mathcal{Z}_{r} \beta_{N}}\left(\frac{M z_{N}}{2 \pi \beta_{N}}\right)^{\frac{1}{2}} \prod_{\alpha=1}^{N} \frac{1}{\beta_{N}\left|\lambda_{\alpha}\right|}
$$

where $z_{N}=\sum_{\alpha}\left(\tilde{\mathbf{R}}_{\alpha}-\tilde{\mathbf{R}}_{\alpha+1}\right)^{2}$, and as in Sec. IIE, the ' indicates $\alpha=2$ is excluded from the product.

\section{RESULTS}

We present the MV-RPI for models I, II, and III converged with 256 beads at $\beta=3.25$ a.u for a nonadiabatic case with $\Delta=0.0077$ a.u.. We find that as the driving force increases the number of beads to the left of the crossing also increases as shown by the nuclear MVRPI in Fig. 4. For the electronic MV-RPI, we use the normalized Wigner population estimator to calculate the population of the $\alpha^{\text {th }}$ bead in the $n^{\text {th }}$ state,

$$
\mathcal{P}_{\alpha n}=\left[\mathbf{x}_{\alpha}\right]_{n}^{2} .
$$

We show that the electronic MV-RPI in Fig. 5 , in agree-



FIG. 4. MV-RPI nuclear bead positions as a function of bead number for model I (red dashed line), model II (green dotted line), and model III (blue dot-dashed line) at $\beta=3.25$ a.u. with $\Delta=0.0077$ a.u. (nonadiabatic). The horizontal black line indicates the position at which donor and acceptor states cross. Note that bead positions for models II and III have all been shifted by 0.5 a.u. and 1.0 a.u., respectively, so that the crossing of all the three models coincide. The number of beads to the left of the crossing increases as the driving force increases.

ment with the nuclear RPI, show an increase in the number of beads in the donor state as the driving force increases. Table. II summarizes the key results of the effect of the driving force on the MV-RPI: the ratio $N_{1} / N_{2}$ grows as a function of the driving force, and is independent of the coupling strength. 




FIG. 5. MV-RPI bead populations as a function of bead number in the donor state for model I (red dashed line), model II (green dotted line), and model III (blue dot-dashed line) at $\beta=3.25$ a.u. with $\Delta=0.077$ a.u. (nonadiabatic). In keeping with the nuclear position changes along the instanton path, the number of beads in the donor state increases as the driving force increases.

\begin{tabular}{|c|c|c|c|c|c|c|c|c|c|}
\hline \multicolumn{4}{|c|}{} & \multicolumn{3}{|c|}{ Adiabatic } & \multicolumn{3}{|c|}{ Nonadiabatic } \\
\hline$\epsilon$ & $N_{1}$ & $N_{2}$ & $R_{0}$ & $\bar{R}$ & $\mathbb{P}_{1}$ & $\mathbb{P}_{2}$ & $\bar{R}$ & $\mathbb{P}_{1}$ & $\mathbb{P}_{2}$ \\
\hline 0.0 & 127 & 127 & 0.0 & $10^{-5}$ & 0.50 & 0.50 & $10^{-5}$ & 0.50 & 0.50 \\
\hline 10.0 & 146 & 108 & -0.5 & -0.71 & 0.57 & 0.43 & -0.74 & 0.57 & 0.43 \\
\hline 20.0 & 163 & 91 & -1.0 & -1.34 & 0.63 & 0.37 & -1.45 & 0.65 & 0.35 \\
\hline
\end{tabular}

TABLE II. 256-bead MV-RPI for models I, II and III with $\beta=3.25$ a.u. for $\Delta=6.15$ a.u. (adiabatic) and $\Delta=0.0077$ a.u. (nonadiabatic). We report the number of beads on the donor $\left(N_{1}\right)$ and acceptor $\left(N_{2}\right)$ surfaces excluding the two beads constrained to the crossing. In each case, we also tabulate the position of the nuclear centroid $(\bar{R})$ and beadaveraged donor and acceptor state populations, $\mathbb{P}_{1}$ and $\mathbb{P}_{2}$, respectively. The values of the nuclear variables, and the energies are given in atomic units.

Next, we explore the structure of the MV-RPI for model I in the adiabatic $(\Delta=6.15$ a.u. $)$ and nonadiabatic $(\Delta=0.0077$ a.u. $)$ regimes with $\beta=3.25$ a.u. In keeping with findings from other multistate instanton calculations,$\underline{43}$ we find that the spread of the nonadiabatic nuclear MV-RPI, shown in Fig. 6, is wider than the corresponding adiabatic MV-RPI. This is a consequence of the higher curvature of the barrier in the nonadiabatic limit as shown in Fig. 7 The electronic MV-RPI (donor state populations) are shown in Fig. 8 , The two beads at the crossing have equal population in the donor and acceptor electronic states in both the adiabatic and nonadiabatic models. However, as shown in the inset, the state populations exhibit oscillatory features near the crossing in



FIG. 6. MV-RPI nuclear bead positions as a function of bead number for model I with $\beta=3.25$ a.u. and $\Delta=6.15$ a.u. (adiabatic, blue line), and $\Delta=0.0077$ a.u. (nonadiabatic, red dashed line). The nonadiabatic instanton exhibits a wider spread than the adiabatic instanton in keeeping with lower curvature of the barrier in the latter case. The black horizontal line marks the position at which donor and acceptor state diabats cross.

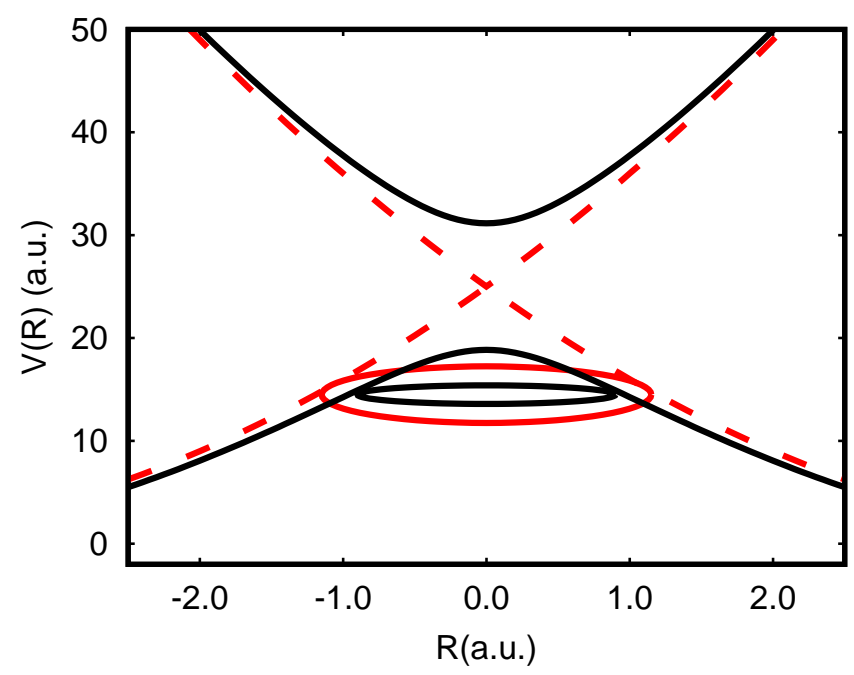

FIG. 7. The adiabatic potential energy surfaces in the adiabatic (black line) and nonadiabatic (red dashed line) coupling limits for model I at $\beta=3.25$ a.u. are shown here. A cartoon repsentation of the extent of spread in the nuclear instanton for both the nonadiabatic (red outer ellipse) and adiabatic (black inner ellipse) limits is also shown.

the adiabatic limit, a feature arising from our use of a diabatic state representation. We also find that while the golden-rule instanton places individual beads on one

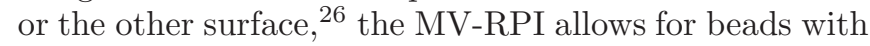
partial populations in keeping with the quantum mechanical probability of the system being in a particular electronic state along the instanton path. 




FIG. 8. electronic MV-RPI donor state populations as a function of bead number for model I with $\beta=3.25$ a.u. and $\Delta=6.15$ a.u. (adiabatic, blue line), and $\Delta=0.0077$ a.u. (nonadiabatic, red dashed line). The inset illustrates electronic state populations vary gradually in the nonadiabatic case but exhibit some oscillatory structure in the adiabatic regime.

Fig. 9 shows the effect of temperature on the nuclear MV-RPI. Specifically, for model I with $\Delta=0.00625$ a.u., we vary $\beta$ from 3.25 a.u. to 4.0 a.u. in steps of 0.25 a.u. We find the nuclear MV-RPI spread increases as temperature decreases; as the system is cooled, we expect a shift towards deep tunneling with a corresponding increase in the extent of the instanton. The electronic MV-RPI does not change significantly over the range of temperatures considered here.

Next, we compare the MF-RPI and the MV-RPI. Given that both are derived from equivalent, exact representations of the quantum canonical partition function, we expect the two nuclear instantons to be nearly indistinguishable as shown in Fig. 10 ,

Although the MF-RPI does not include explicit electronic variables, it is possible to estimate the electronic state populations from the nuclear bead positions along the nuclear instanton path. In keeping with previous work 18 , we calculate the donor state population of each bead using the following ad hoc expression,

$$
\mathbb{P}_{\alpha 1}=\frac{e^{-\beta V_{11}\left(\mathbf{R}_{\alpha}\right)}}{e^{-\beta V_{11}\left(\mathbf{R}_{\alpha}\right)}+e^{-\beta V_{22}\left(\mathbf{R}_{\alpha}\right)}}
$$

We compare this MF-RPI result against the electronic MV-RPI populations in Fig. 11(a) for nonadiabatic model I with $\Delta=0.001$ a.u and $\beta=3.25$ a.u.. Examining beads in the vicinity of the crossing, shown in Fig. 11(b) and Fig. 11(c), we find that populations obtained from the nuclear MF-RPI fail to distinguish between the adiabatic and nonadiabatic regimes over three orders of magnitude. However, the electronic MV-RPI populations show a smooth transition from beads on one

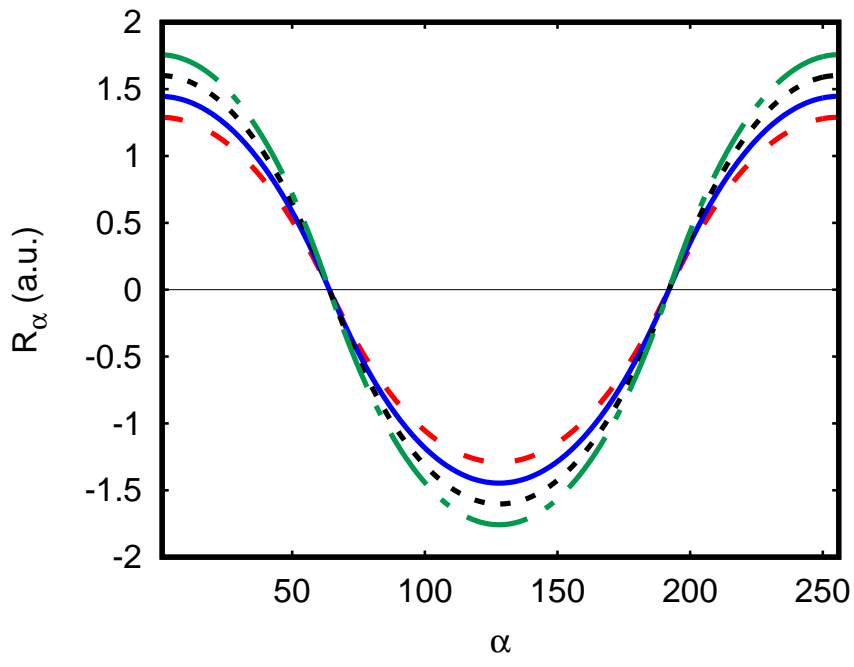

FIG. 9. Nucelar bead positions as a function of bead number for a 256-bead MV-RPI for model I with $\Delta=0.00625$ a.u. (nonadiabatic) and $\beta=3.25,3.5,3.75,4.0$ a.u. represented by red dashed, blue continuous, black dotted, and green dotdashed lines, respectively. The horizontal line is the crossing of the donor and acceptor diabats.

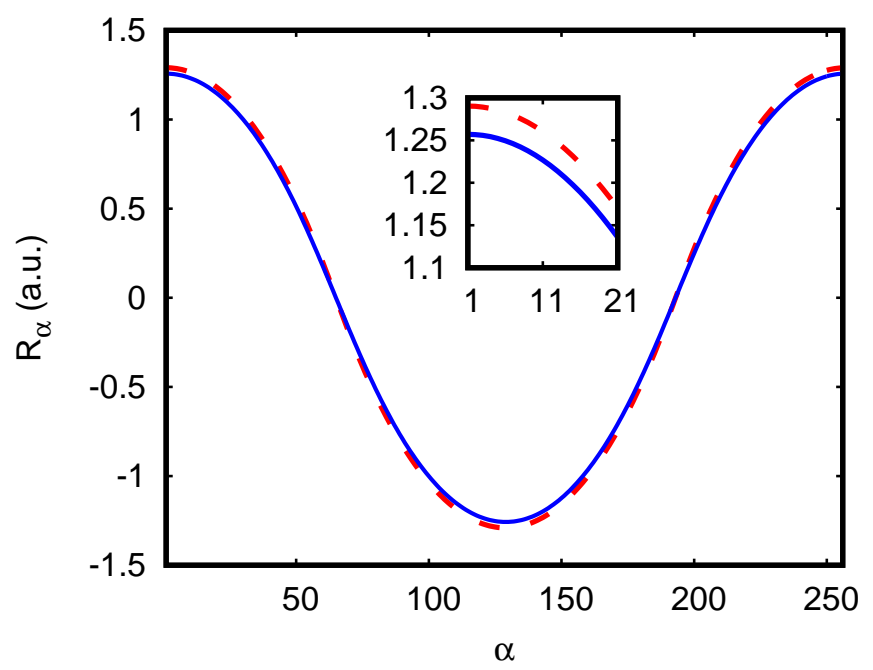

FIG. 10. Nuclear bead positions as a function of bead number for a 256-bead MV-RPI determined for model I at $\beta=3.25$ a.u. in the nonadiabatic limit $(\Delta=0.0077$ a.u. $)$. The MFRPI nuclear positions are represented using the blue line while those obtained from the MV-RPI using the red dotted lines. The inset highlights nuclear positions for the first 20 beads where the small numerical difference between the two instantons is most noticeable.

state to the other along the instanton path in the nonadiabatic case and an oscillatory structure in the adiabatic case (as expected when working in the diabatic representation). We also find that in the adiabatic regime, beads away from the crossing are not fully in one or the other diabatic state in keeping with the underlying model.

Finally, we calculate MF-RPI rate constants. In 

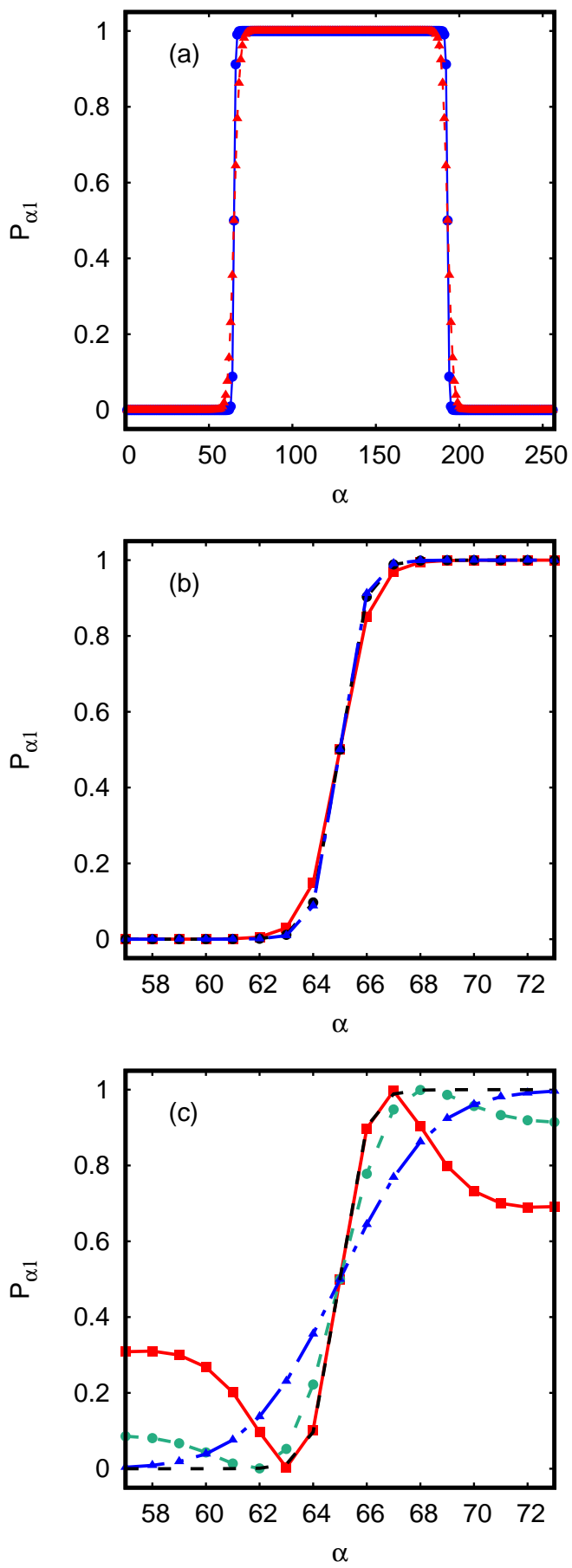

FIG. 11. (a) Comparing donor state populations obtained from the nuclear MF-RPI instanton (blue line with circles) against the electronic MV-RPI populations (red dashed line with triangles) for model I with $\Delta=0.001$ a.u. and $\beta=3.25$ a.u. (b) Populations for beads in the vicinity of the crossing obtained from the nuclear MF-RPI instanton for model I with $\beta=3.25$ a.u. shown for three different coupling strengths, $\Delta=6.15$ a.u (red line with squares), $\Delta=2.5$ a.u (black dashed line with triangles), and $\Delta=0.001$ a.u (blue dot-dashed line with circles). (c) Electronic MV-RPI populations for beads in the vicinity of the crossing for model I with $\beta=3.25$ a.u. for three different coupling strengths, $\Delta=6.15$ a.u (red line with squares), $\Delta=2.5$ a.u (green dashed line with circles), and $\Delta=0.001$ a.u (blue dot-dashed line with triangles). For comparison, the MF-RPI populations are shown for $\Delta=2.5$ a.u. (black dashed line).

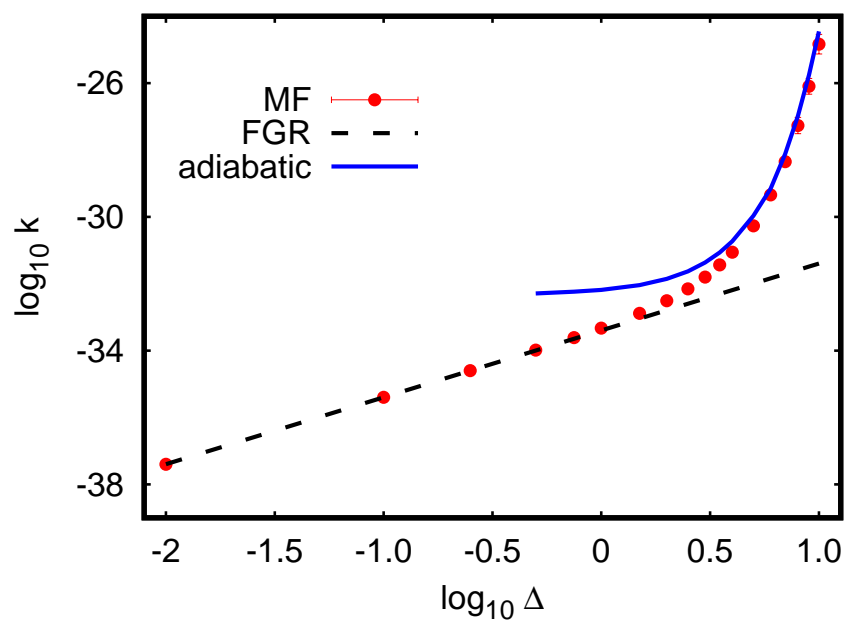

FIG. 12. MF-RPI rate constants (red circles) as a function of the coupling strength for model I at $\beta=4.0$ a.u. In the nonadiabatic regime, we find good agreement with the FGR rate constants (dashed black line) and in the adiabatic regime, MF-RPI results are within a factor of 2 of the single surface RPI rate constants (blue line).

Fig. 12 we present results for model I as a function of the electronic coupling, $\Delta$. In the nonadiabatic regime, we find the MF-RPI rate constants are in good agreement with FGR rate constants. As we approach the adiabatic, strong coupling regime where FGR is not applicable, we calculate the single surface RPI rate on the lower adiabatic surface and find that they agree well with the MFRPI rates, as reported in Table III. In the intermediate coupling regime, we find the MF-RPI rate expression interpolates smoothly and accurately between the nonadiabatic and adiabatic dynamic regimes.

\begin{tabular}{|c|c|c|c|}
\hline$\Delta$ & $\log _{10} k_{\mathrm{MF}-\mathrm{RPI}}$ & $\log _{10} k_{\mathrm{FGR}}$ & $\log _{10} k_{R P}$ \\
\hline 0.01 & -37.398 & -37.395 & - \\
0.1 & -35.397 & -35.395 & - \\
0.25 & -34.598 & -34.599 & - \\
1.0 & -33.327 & -33.395 & -32.182 \\
2.5 & -32.153 & -32.599 & -31.628 \\
5.0 & -30.269 & -31.997 & -29.966 \\
10.0 & -24.836 & -31.395 & -24.444 \\
\hline
\end{tabular}

TABLE III. Comparing rate constants for model I at $\beta=4.0$ a.u. with different coupling strengths. $k_{\mathrm{MF}-\mathrm{RPI}}$ is the MF$\mathrm{RPI}$ rate constant, $k_{\mathrm{FGR}}$ is the rate constant obtained using Fermi's Golden Rule, and $k_{\mathrm{RP}}$ is the rate constant computed using single surface RPI determined on the lower adiabatic surface. Note that $k_{\mathrm{RP}}$ is only calculated for cases where the RPI has a zero mode.

We also find good agreement between MF-RPI rates and FGR rates for models I, II, and III in nonadiabatic 
regime with $\Delta=0.0625$ a.u. where driving force is varied as reported in Table IV

\begin{tabular}{|c|c|c|c|}
\hline Model & $\epsilon$ & $\log _{10} k_{\mathrm{MF}-\mathrm{RPI}}$ & $\log _{10} k_{\mathrm{FGR}}$ \\
\hline I & 0.0 & -37.805 & -37.803 \\
II & 10.0 & -29.913 & -29.913 \\
III & 20.0 & -23.485 & -23.508 \\
\hline
\end{tabular}

TABLE IV. MF-RPI rates compared with FGR rates for nonadiabatic models I, II and III with $\beta=4.0$ a.u., $\Delta=$ 0.0625 a.u.

\section{CONCLUSION}

In this paper, we compute multistate RPIs for nonadiabatic systems that are valid in the adiabatic regime as well. We show that, as expected, both the MF and MV representations arrive at identical nuclear instantons, but the electronic MV-RPI uniquely reports on electronic state transitions along the instanton path. We obtain analytic expressions for the zero-mode in both cases and numerically establish that all solutions reported here correspond to a first order saddle. We find that the electronic MV-RPI shows a gradual change in population of beads from donor to acceptor state in the nonadiabatic case and a more steep change in the adiabatic case along with some oscillatory structure. We clearly demonstrate that electronic state populations obtained from the nuclear MF-RPI fail to distinguish between the adiabatic and nonadiabatic regimes. We also note that the electronic MV-RPI captures beads with fractional state population in keeping with the quantum mechanical probability of being in a given electronic state, unlike instantons based on Fermi's golden rule that place beads on one or the other surface.

Finally, we obtain an expression for the MF-RPI rate and demonstrate that it is accurate for the calculation of rates in both the adiabatic and nonadiabatic regimes. We expect this will prove a powerful tool for rapid rate calculations. We do not provide a separate MV-RPI rate equation since the MF and MV path integral expressions are equivalent (and exact) for the calculation of statistical properties, and as such can be reasonably expected to yield numerically equivalent instanton rates in all regimes. Rather, we view the MV-RPI as an essential step towards characterizing the mechanism of nonadiabatic dynamic processes through its connection with an optimal dividing surface transition state theory ${ }^{15}$ and as a way to initialize trajectories in MV-RPMD simulations of multistate population dynamics.

\section{ACKNOWLEDGMENTS}

The authors would like to thank Prof. Ezra and Prof. DiStasio for helpful discussions. This work was funded through a National Science Foundation CAREER grant (Award No. CHE-1555205).

\section{Appendix A ZERO MODE OF THE MF INSTANTON}

We obtain the MF action from the continuous $(N \rightarrow$ $\infty)$ limit of the effective MF ring polymer potential

$$
\begin{aligned}
\mathcal{S}_{\mathrm{MF}} & =\lim _{N \rightarrow \infty} \beta_{N} V_{\mathrm{MF}} \\
& =\lim _{N \rightarrow \infty} \beta_{N}\left[\frac{M}{2 \beta_{N}^{2}} \sum_{\alpha}\left(\mathbf{R}_{\alpha}-\mathbf{R}_{\alpha+1}\right)^{2}-\frac{1}{\beta_{N}} \ln \Gamma_{\mathrm{MF}}\right] \\
& =\int_{0}^{\beta} d \tau\left[\frac{M}{2}\left(\frac{d \mathbf{R}(\tau)}{d \tau}\right)^{2}-\frac{d \ln \left|\Gamma_{\mathrm{MF}}[\mathbf{R}(\tau)]\right|}{d \tau}\right] \\
& =\int_{0}^{\beta} d \tau\left[\frac{M}{2}\left(\frac{d \mathbf{R}(\tau)}{d \tau}\right)^{2}+\mathcal{V}_{\mathrm{MF}}\right]
\end{aligned}
$$

Here, we define $-\frac{d \ln \left|\Gamma_{\mathrm{MF}}[\mathbf{R}(\tau)]\right|}{d \tau} \equiv \mathcal{V}_{\mathrm{MF}}$ for clarity of presentation. Note that in obtaining Eq. 35, we used the finite difference definition of a derivative for the nuclear term and we introduce the integral of a differential operator in $\tau$ for the $\ln \left|\Gamma_{\mathrm{MF}}\right|$ term. Setting the first variation of the action to zero, we obtain Newton's equations in imaginary time on the inverted potential,

$$
\delta \mathcal{S}_{\mathrm{MF}}=-M \frac{d^{2} \mathbf{R}(\tau)}{d \tau^{2}}+\nabla_{\mathbf{R}} \mathcal{V}_{\mathrm{MF}}=0
$$

The MF instanton is the solution to these $N \times f$ equations.

The instanton is an unstable periodic orbit, and this is established by calculating the eigenvalues of the stability matrix. We obtain the stability matrix from the second variation of the action $\delta^{2} \mathcal{S}_{\mathrm{MF}}$,

$$
\Lambda_{\mathrm{MF}} \equiv-M \frac{d^{2}}{d \tau^{2}}+\nabla_{\mathbf{R}} \nabla_{\mathbf{R}}^{T} \mathcal{V}_{\mathrm{MF}}
$$

Differentiating Eq. 36 with respect to imaginary time, we obtain

$$
\begin{aligned}
& \frac{d}{d \tau}\left[-M \frac{d^{2} \mathbf{R}(\tau)}{d \tau^{2}}+\nabla_{\mathbf{R}} \mathcal{V}_{\mathrm{MF}}\right]=0 \\
& {\left[-M \frac{d^{2}}{d \tau^{2}}+\nabla_{\mathbf{R}} \nabla_{\mathbf{R}}^{T} \mathcal{V}_{\mathrm{MF}}\right] \dot{\mathbf{R}}(\tau)=0 \times \dot{\mathbf{R}}(\tau)}
\end{aligned}
$$

It is clear that Eq. 38 is the eigenvalue equation corresponding to operator $\Lambda_{\mathrm{MF}}$ and the zero-mode is the velocity mode, $\dot{\mathbf{R}}(\tau)$, with a zero eigenvalue. 


\section{Appendix B ZERO MODE OF THE MV INSTANTON}

The action $\mathcal{S}_{\mathrm{MV}}$ is the imaginary-time integral of the Langrangian $\mathcal{L}_{\mathrm{MV}}$ given by

$$
\mathcal{L}_{\mathrm{MV}}=\left[\begin{array}{c}
\frac{M}{2}\left(\frac{d \mathbf{R}(\tau)}{d \tau}\right)^{T}\left(\frac{d \mathbf{R}(\tau)}{d \tau}\right)+\frac{d}{d \tau} \mathbf{x}(\tau)^{T} \mathbf{x}(\tau) \\
-\frac{d}{d \tau} \ln |\Gamma[\mathbf{R}(\tau), \mathbf{x}(\tau)]|
\end{array}\right]
$$

Following our treatment of the MF action, we define

$$
\mathcal{V}_{\mathrm{MV}} \equiv-\frac{d}{d \tau} \ln |\Gamma[\mathbf{R}(\tau), \mathbf{x}(\tau)]|
$$

The first variation of action yields a set of coupled equations of motion for the nuclear and electronic variables,

$$
\begin{aligned}
\delta \mathcal{S}_{\mathrm{MV}} & =\int d \tau \delta \mathbf{R}(\tau)\left[-M\left(\frac{d^{2} \mathbf{R}(\tau)}{d \tau^{2}}\right)+\nabla_{\mathbf{R}} \mathcal{V}_{\mathrm{MV}}\right] \\
& +\int d \tau \delta \mathbf{x}(\tau)\left[-\nabla_{\mathbf{x}} \mathcal{V}_{\mathrm{MV}}\right]
\end{aligned}
$$

The second variation of action gives,

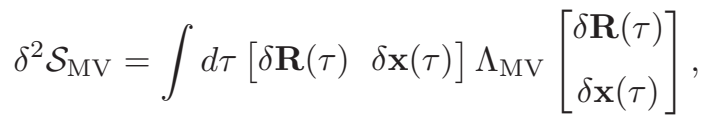

where $\Lambda_{\mathrm{MV}}$ is the $(f+\mathcal{K}) \times(f+\mathcal{K})$ dimensional stability matrix,

$$
\left[\begin{array}{cc}
-M\left(\frac{d^{2}}{d \tau^{2}}\right)+\nabla_{\mathbf{R}} \nabla_{\mathbf{R}}^{T} \mathcal{V}_{\mathrm{MV}} & -\nabla_{\mathbf{R}} \nabla_{\mathbf{x}}^{T} \mathcal{V}_{\mathrm{MV}} \\
-\nabla_{\mathbf{x}} \nabla_{\mathbf{R}}^{T} \mathcal{V}_{\mathrm{MV}} & -\nabla_{\mathbf{x}} \nabla_{\mathbf{x}}^{T} \mathcal{V}_{\mathrm{MV}}
\end{array}\right]
$$

Differentiating Eq.40, we once again obtain an eigenvalue equation for the stability operator,

$$
\Lambda_{\mathrm{MV}}\left[\begin{array}{c}
\dot{\mathbf{R}}(\tau) \\
\dot{\mathbf{x}}(\tau)
\end{array}\right]=\left[\begin{array}{l}
0 \\
0
\end{array}\right]=0\left[\begin{array}{c}
\dot{\mathbf{R}}(\tau) \\
\dot{\mathbf{x}}(\tau)
\end{array}\right] .
$$

It is clear from Eq. 42 that the collective velocity mode (in both nuclear and electronic variables) is the zero mode.

\section{REFERENCES}

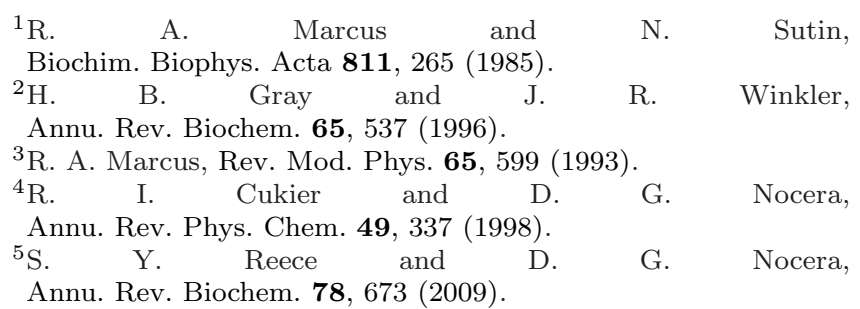

${ }^{6}$ W. H. Miller, J. Chem. Phys. 62, 1899 (1975)

${ }^{7}$ J. S. Langer, Ann. Phys. 281, 941 (2000).

${ }^{8}$ S. Coleman, "The uses of instantons," in The Whys of Subnuclear Physics (Springer US, Boston, MA, 1979) pp. 805-941.

${ }^{9}$ S. Chapman, B. C. Garrett, and W. H. Miller, J. Chem. Phys. 63, 2710 (1975)

${ }^{10}$ W. H. Miller, S. D. Schwartz, and J. W. Tromp, J. Chem. Phys. 79, 4889 (1983).

${ }^{11}$ C. G. Callan and S. Coleman, Phys. Rev. D 16, 1762 (1977)

${ }^{12}$ S. Coleman, Phys. Rev. D 15, 2929 (1977)

${ }^{13}$ M. Kryvohuz, J. Chem. Phys. 134, 114103 (2011)

${ }^{14}$ W. H. Miller, Y. Zhao, M. Ceotto, and S. Yang, J. Chem. Phys. 119, 1329 (2003).

${ }^{15}$ J. O. Richardson and S. C. Althorpe, J. Chem. Phys. 131, 214106 (2009)

${ }^{16}$ S. C. Althorpe, J. Chem. Phys. 134, 114104 (2011)

${ }^{17}$ J. O. Richardson, J. Chem. Phys. 144, 114106 (2016).

${ }^{18}$ C. D. Schwieters and G. A. Voth, J. Chem. Phys. 108, 1055 (1998).

${ }^{19}$ C. D. Schwieters and G. A. Voth, J. Chem. Phys. 111, 2869 (1999).

${ }^{20}$ S. Jang and J. Cao, J. Chem. Phys. 114, 9959 (2001)

${ }^{21}$ P. G. Wolynes, J. Chem. Phys. 87, 6559 (1987).

${ }^{22}$ J. E. Lawrence and D. E. Manolopoulos, J. Chem. Phys. 148, 102313 (2018).

${ }^{23}$ M. C. Gutzwiller, J. Math. Phys. 8, 1979 (1967).

${ }^{24}$ M. C. Gutzwiller, J. Math. Phys. 12, 343 (1971).

${ }^{25}$ J. O. Richardson, R. Bauer, and M. Thoss, J. Chem. Phys. 143, 134115 (2015).

${ }^{26}$ J. O. Richardson, J. Chem. Phys. 143, 134116 (2015).

${ }^{27}$ G. Stock and M. Thoss, Phys. Rev. Lett. 78, 578 (1997)

${ }^{28}$ H. Meyer and W. H. Miller, J. Chem. Phys. 70, 3214 (1979)

${ }^{29}$ N. Ananth and T. F. Miller III, J. Chem. Phys. 133, 234103 (2010).

${ }^{30}$ P. Huo and D. F. Coker, J. Chem. Phys. 135, 201101 (2011).

${ }^{31}$ A. Kelly, R. van Zon, J. Schofield, and R. Kapral, J. Chem. Phys. 136, 084101 (2012).

${ }^{32}$ N. Ananth, J. Chem. Phys. 139, 124102 (2013)

${ }^{33} \mathrm{~S}$ N. Chowdhury and P. Huo, J. Chem. Phys. 147, 214109 (2017)

${ }^{34}$ W. H. Miller and S. J. Cotton, Farad. Discuss. 195, 9 (2017).

${ }^{35}$ C. Zhu, R. H. Byrd, P. Lu, and J. Nocedal, ACM Transactions on Mathematical Software (TOMS) 23, 550 (1997).

${ }^{36} \mathrm{~J}$ O. Richardson and M. J. Chem. Phys. 139, 31102 (2013)

37 J. R. Duke and N. Ananth, Farad. Discuss. 195, 253 (2017)

38 J. Schmidt and J. C. Tully, J. Chem. Phys. 127, 094103 (2007).

${ }^{39}$ T. J. H. Hele, (2013) An Electronically Non-Adiabatic Generalization of Ring Polymer Molecular Dynamics, MChem thesis, Exeter College, University of Oxford, 2011, arXiv:1308.3950

${ }^{40} \mathrm{~S}$. Pierre, J. R. Duke, T. J. H. Hele, and N. Ananth, J. Chem. Phys. 147, 234103 (2017)

${ }^{41}$ H.-D. Meyer and W. H. Miller, J. Chem. Phys. 71, 2156 (1979)

${ }^{42} \mathrm{~V}$. A. Benderskii, D. E. Makarov, and C. A. Wight, Chemical dynamics at low temperatures (Wiley, 1994) p. 385.

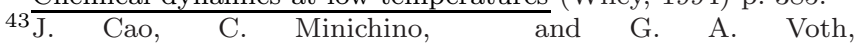
J. Chem. Phys. 103, 1391 (1995) 